\title{
UNA NUEVA ESPECIE DE DIOSCOREA (DIOSCOREACEAE) PARA LAS YUNGAS DEL NOROESTE ARGENTINO
}

\author{
Patricia N. Asesor (iD \& Eva Bulacio \\ Laboratorio de Taxonomía Fanerogámica y Palinología, Fundación Miguel Lillo. Miguel Lillo 251, T4000JFE San \\ Miguel de Tucumán, Tucumán, Argentina; pnasesor@lillo.org.ar (autora corresponsal).
}

\begin{abstract}
Asesor, P. N. \& E. Bulacio. 2021. A new species of Dioscorea (Dioscoreaceae) for the Yungas of northwestern Argentina. Darwiniana, nueva serie 9(2): 393-400.

A new species of Dioscorea endemic to the Yungas of the northwestern Argentina, is here described as Dioscorea ayardei. This new species is characterized by the presence of a single anther inserted in a navicular structure with three dendriform projections. The new species is morphologically similar to $D$. haumanii and both inhabit mountain areas at the Sierras of Calilegua in the Jujuy province. A description of $D$. ayardei, together with illustrations, a distribution map, habitat description, conservation assessment, a comparative table with the related species and a diagnostic comparison with $D$. haumanii is presented.
\end{abstract}

Keywords. Argentine Yungas; Dioscorea; Sierras de Calilegua; taxonomy.

Resumen. Asesor, P. N. \& E. Bulacio. 2021. Una nueva especie de Dioscorea (Dioscoreaceae) para las Yungas del noroeste argentino. Darwiniana, nueva serie 9(2): 393-400.

Una nueva especie de Dioscorea, endémica de las Yungas del noroeste de Argentina, es aquí descripta como Dioscorea ayardei. Esta nueva especie se caracteriza por la presencia de una única antera inserta en una estructura navicular con tres proyecciones dendriformes. La nueva especie es morfológicamente afín a $D$. haumanii y ambas habitan las áreas montanas de las Sierras de Calilegua en la provincia de Jujuy. Se presenta una descripción de $D$. ayardei, ilustraciones, un mapa de distribución, descripción del hábitat, evaluación del estado de conservación, un cuadro comparativo con las especies afines y la comparación del diagnóstico con $D$. haumanii.

Palabras clave. Dioscorea; Sierras de Calilegua; taxonomía; Yungas argentinas.

\section{INTRODUCCIÓN}

Dioscorea L. (Dioscoreaceae) es un género de distribución mundial, que cuenta con alrededor de 650 especies que habitan las regiones templadas y tropicales, especialmente en el Neotrópico (Couto et al., 2018). En Argentina, el género se distribuye principalmente en el norte, este y unas pocas especies en el sur del país (Asesor et al., 2019) $\mathrm{y}$ se reconocen un total de 33 entidades, de las cuales 11 son endémicas (Zuloaga et al., 2021) la mayoría distribuidas a lo largo del área andina. Las especies de Dioscorea son en su mayoría enredaderas, monoicas o dioicas, generalmente con flores pequeñas y con tubérculos ricos en almidón como órgano subterráneo. El valor nutricional de su sistema subterráneo caracteriza a muchas especies del género, siendo una importante fuente de energía y de gran valor económico para poblaciones humanas en todo 
el mundo debido a los metabolitos secundarios presentes en esas estructuras (Coursey, 1967; Wilkin et al., 2005). Como consecuencia de la gran variedad de condiciones ambientales en las que ocurren, las especies exhiben una amplia gama de respuestas ecológicas, que se reflejan en la gran variabilidad morfológica encontrada en la familia en los órganos vegetativos y reproductivos (Couto et al., 2018).

En el transcurso del estudio, sobre el género Dioscorea de las montañas del noroeste argentino, llevado a cabo en las Sierras de Calilegua (Jujuy, Argentina), colectamos especímenes del género que no pudimos adjudicarlos a ninguna especie conocida de Argentina. Nuevas colectas y observaciones "in situ" en distintas localidades de las mencionadas sierras, consultas a herbarios de referencia de Argentina, Bolivia, Perú y a herbarios virtuales, sumado a los análisis de rutina de caracteres morfológicos del material revisado, aportaron información suficiente para proponerla como una nueva especie. En este artículo se da a conocer el hallazgo de Dioscorea ayardei Asesor una especie que crece en las montañas del noroeste de Argentina.

\section{MATERIALES Y MÉTODOS}

El material estudiado proviene de las Sierras de Calilegua, Jujuy, las que se caracterizan por una marcada estacionalidad climática, con precipitaciones que ocurren en verano y varían de acuerdo con la altitud, alcanzando valores de entre 1.000 a $2.000 \mathrm{~mm}$ al año (Ayarde, 2013). La vegetación, típica de la provincia fitogeográfica de las Yungas (Cabrera, 1976) o selva de las Yungas (Morello et al., 2012), se dispone en fajas altitudinales desde el bosque deciduo pedemontano hasta los bosques y pastizales montanos (Bulacio \& Ayarde, 2009; Ayarde, 2013) (Fig. 1).

$\mathrm{Se}$ examinaron especímenes y material tipo de los siguientes herbarios: BA, BAF, BOLV, CORD, HSB, HSP, LIL, LPB, MCNS, SI, USZ, USM y herbarios virtuales (ASU, B, BR, F, FURB, HUEFS, INCT, K, MBM, MBML, NY, UPCB, US) (Thiers, 2021), tanto de la nueva especie como de las especies afines presentes en la Argentina y en países limítrofes próximos a las zonas de hallazgo (Tabla 1).
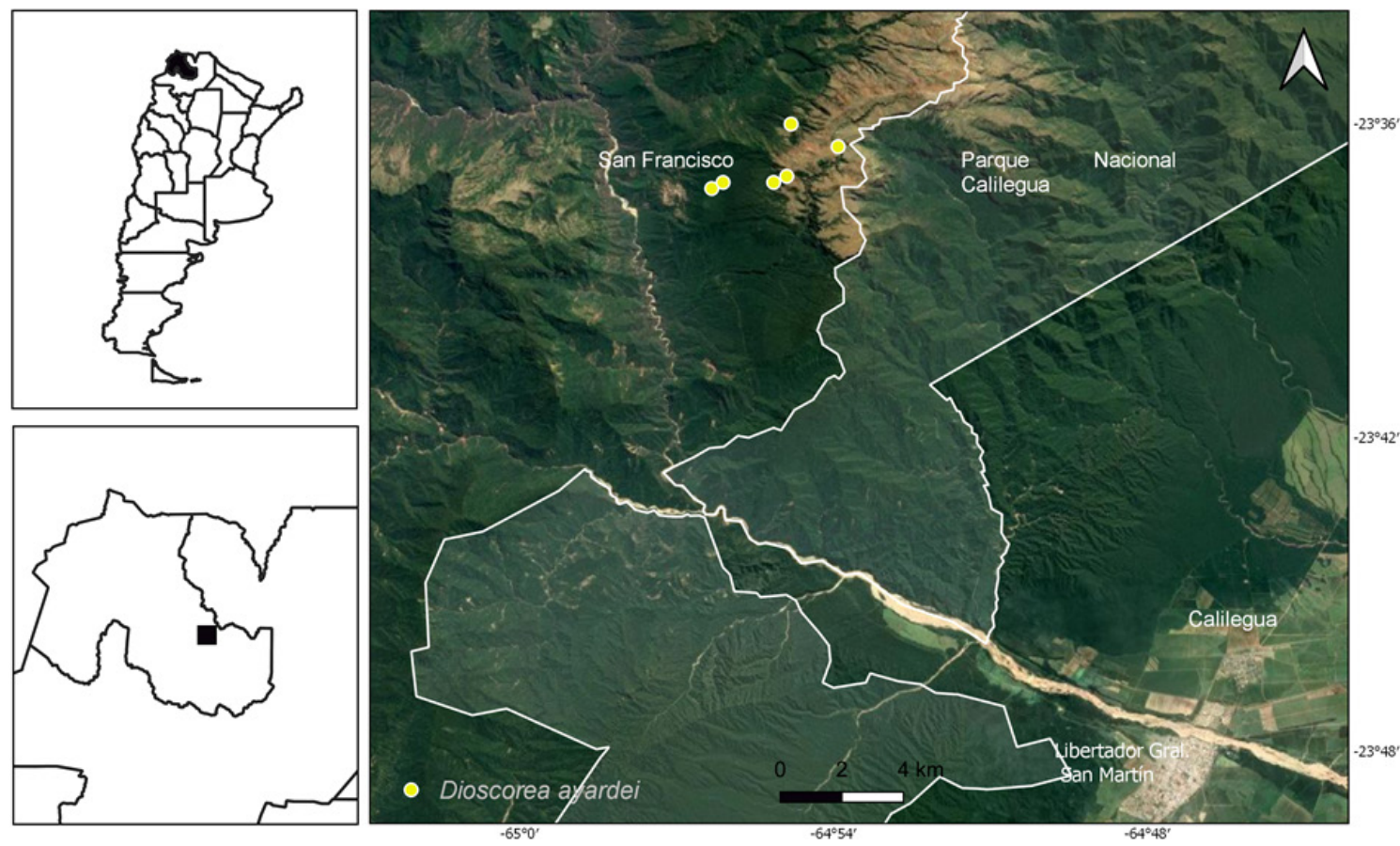

Fig. 1. Distribución geográfica de Dioscorea ayardei. Figura en color en la versión en línea http://www.ojs.darwin.edu. ar/index.php/darwiniana/article/view/985/1239 
Luego del análisis detallado de los ejemplares, se estudiaron imágenes y literatura de las especies del género. Las descripciones se basaron en material fresco que se fijó en solución conservante (FAA) mientras que otros fueron herborizados y serán depositados en el Herbario de la Fundación Miguel Lillo (LIL).

\section{RESULTADOS}

\section{Tratamiento taxonómico}

Dioscorea ayardei Asesor sp. nov. TIPO: ARGENTINA, Jujuy, Valle Grande, Sierras de Calilegua, senda de San Francisco a Alto Calilegua, $1 \mathrm{~km}$ antes de Duraznillo, 24-II2015, P. Asesor, E. Bulacio, H. Ayarde \& S. Buedo, As. 113 (Holotipo, LIL). (Figs. 2 y 3).

Climbing herb, diclino-monoecious. Twining vine-dextrorse stem, more than $2 \mathrm{~m}$; spherical tubercle, 3-5 cm diam. Sheets ovate-triangular or sagittate, with a narrow basal sinus of $0.5-2.3 \mathrm{~cm}$, acute or acuminate apex, mucronate, cordate or sub-cordate base; petiole 2-4 cm. Racemose staminate inflorescence, $5-10 \mathrm{~cm}$, with (8) 9 or 10 flowers; peduncle 10-15 mm. Solitary flowers; pedicel 2-3 cm; perianth stellate, 5-6 $\times$ 1.5-2 mm, purple with greenish-white edges; short staminal column, ca. $1 \mathrm{~mm}$, widened in its apical part into a navicular body, 3.5$4.5 \times 1.5-2 \mathrm{~mm}$, finished in three dendriform projections, the central purple and the lateral greenish-white, bearded; single anther inserted in the center of the navicular body; null style rudiment. Pistillate inflorescences in spike, $6-10 \mathrm{~cm}$; flowers purple, $1-1.2 \times 0.4-0.5 \mathrm{~mm}$, style with three stigmatic branches, $0.5-0.8 \mathrm{~mm}$; oblong ovary, $1.8-2 \times 1-1.2 \mathrm{~mm}$. Trialated capsule, $1-1.1 \times 0.5-0.6 \mathrm{~cm}$. Seeds 2-3 × 1-2 mm.

Hierba trepadora, diclino-monoica. Tallo voluble-dextrorso subcilíndrico de más de $2 \mathrm{~m}$, glabro, estriado; tubérculo esférico cubierto de raíces cortas, 3-5 cm de diám. Hojas membranáceas; láminas ovado-triangulares o sagitadas, con seno basal estrecho de 0,5-2,3 cm separando lobos redondeados, ápice agudo o acuminado, mucronado, base cordada o sub-cordada, con 7-8 nervaduras, las laterales 2-3 furcadas, $6-10 \times 3,5-4 \mathrm{~cm}$, superficie abaxial y adaxial pubescentes; pecíolo de 2-4 cm, pubescente. Inflorescencias estaminadas racemosas, 1 por axila, de $5-10 \mathrm{~cm}$, laxas (8)9 o 10 flores; pedúnculo de 10$15 \mathrm{~mm}$, pubérulo; bractéolas de 1,5-2 $\mathrm{mm}$, lanceoladas, membranáceas. Flores solitarias; pedicelo de $2-3 \mathrm{~cm}$; perianto estrellado, tépalos subiguales, $5-6 \times 1,5-2 \mathrm{~mm}$, purpúreos en el centro con los bordes blanco-verdosos, los externos angostamente ovados y los internos oval-lanceolados, trinervados, las nervaduras laterales más cortas que la central, con abundantes pelos eglandulares en la cara adaxial; columna estaminal corta, ca. $1 \mathrm{~mm}$, ensanchada en su parte apical en un cuerpo navicular de 3,5-4,5 $\times 1,5-2 \mathrm{~mm}$, terminado en tres proyecciones dendriformes, la central púrpura y las laterales blanco-verdosas, barbadas; antera única, con línea de dehiscencia evidente inserta en el centro del cuerpo navicular; rudimento estilar nulo. Inflorescencias pistiladas en espiga, pubérulas, de $6-10 \mathrm{~cm}$; flores purpúreas casi perpendiculares al raquis; tépalos oblongos con ápice obtuso, 1-1,2 × 0,4$0,5 \mathrm{~mm}$, estilo con tres ramas estigmáticas de 0,5-0,8 mm; ovario oblongo, 1,8-2 × 1-1,2 mm. Cápsula trialada, oblonga, con restos de perianto en el extremo, $1-1,1 \times 0,5-0,6 \mathrm{~cm}$. Semillas 2-3 $\times 1-2 \mathrm{~mm}$, con un ala membranosa en su extremo inferior, $2-4 \times 1,3-1,5 \mathrm{~mm}$.

Etimología. El epíteto específico está dedicado al botánico Hugo Ramón Ayarde, de la Fundación Miguel Lillo, por su valioso aporte al estudio de la flora de las Sierras de Calilegua.

Distribución y hábitat. Dioscorea ayardei se restringe a las áreas montanas de las Sierras de Calilegua, en el nivel superior del bosque montano y arbustal mesofítico, en bordes de bosques, quebradas húmedas y pastizales entre los 1.500 y $2.800 \mathrm{~m} \mathrm{s.m}$. Crece sobre laderas de areniscas o sobre arbustos, compartiendo hábitat con otras especies como Berberis commutata Eichler. (Berberidaceae), Baccharis spp. (Asteraceae), Solanum spp. (Solanaceae), 


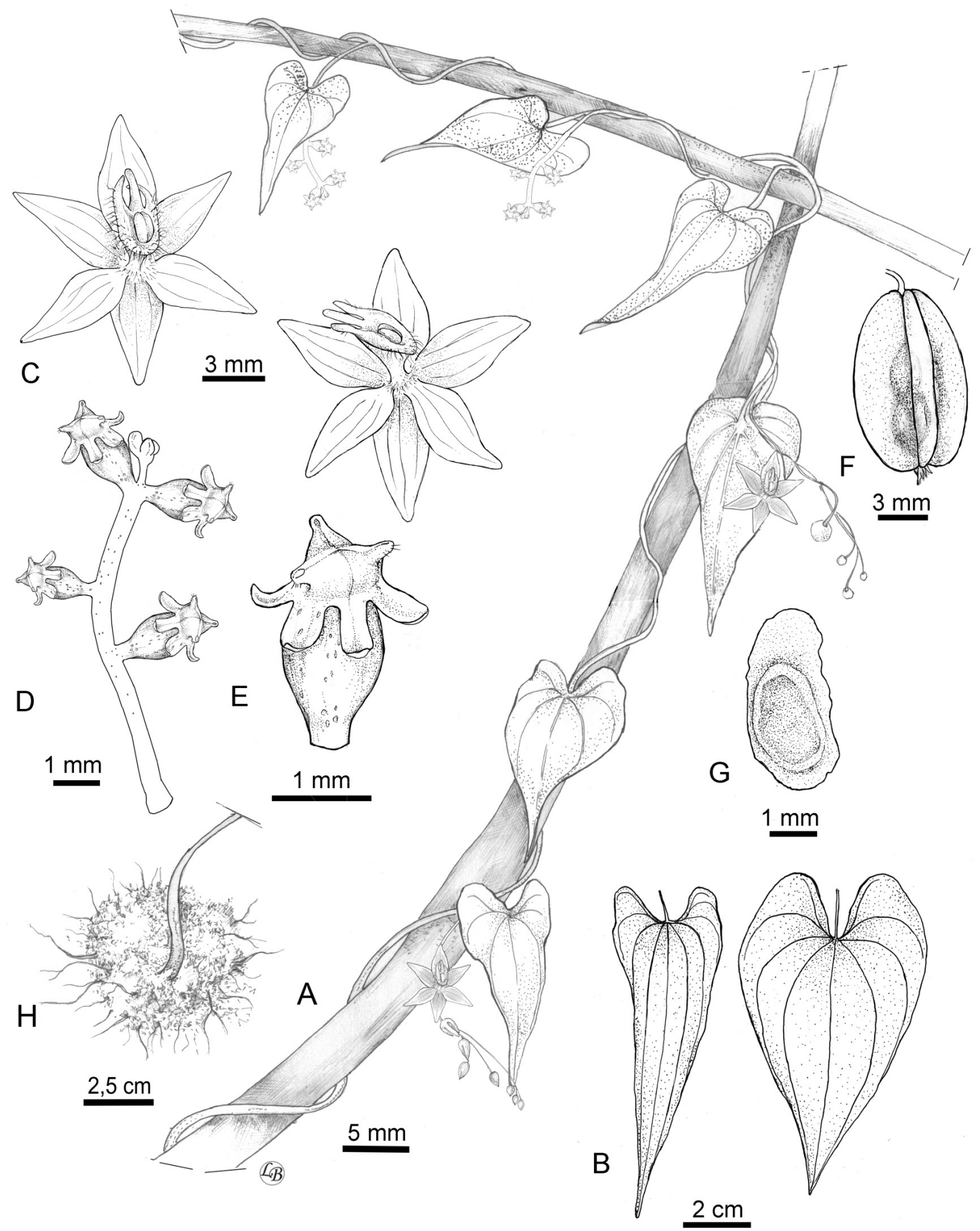

Fig. 2. Dioscorea ayardei. A, aspecto general de la planta. B, tipos de hojas. C, flor estaminada. D, espiga femenina. E, flor pistilada. F, fruto. G, semilla. H, tubérculo. A-C-D-E-F-G-H de Asesor et al. 113 (LIL); B de Asesor et al. 115 (LIL). 

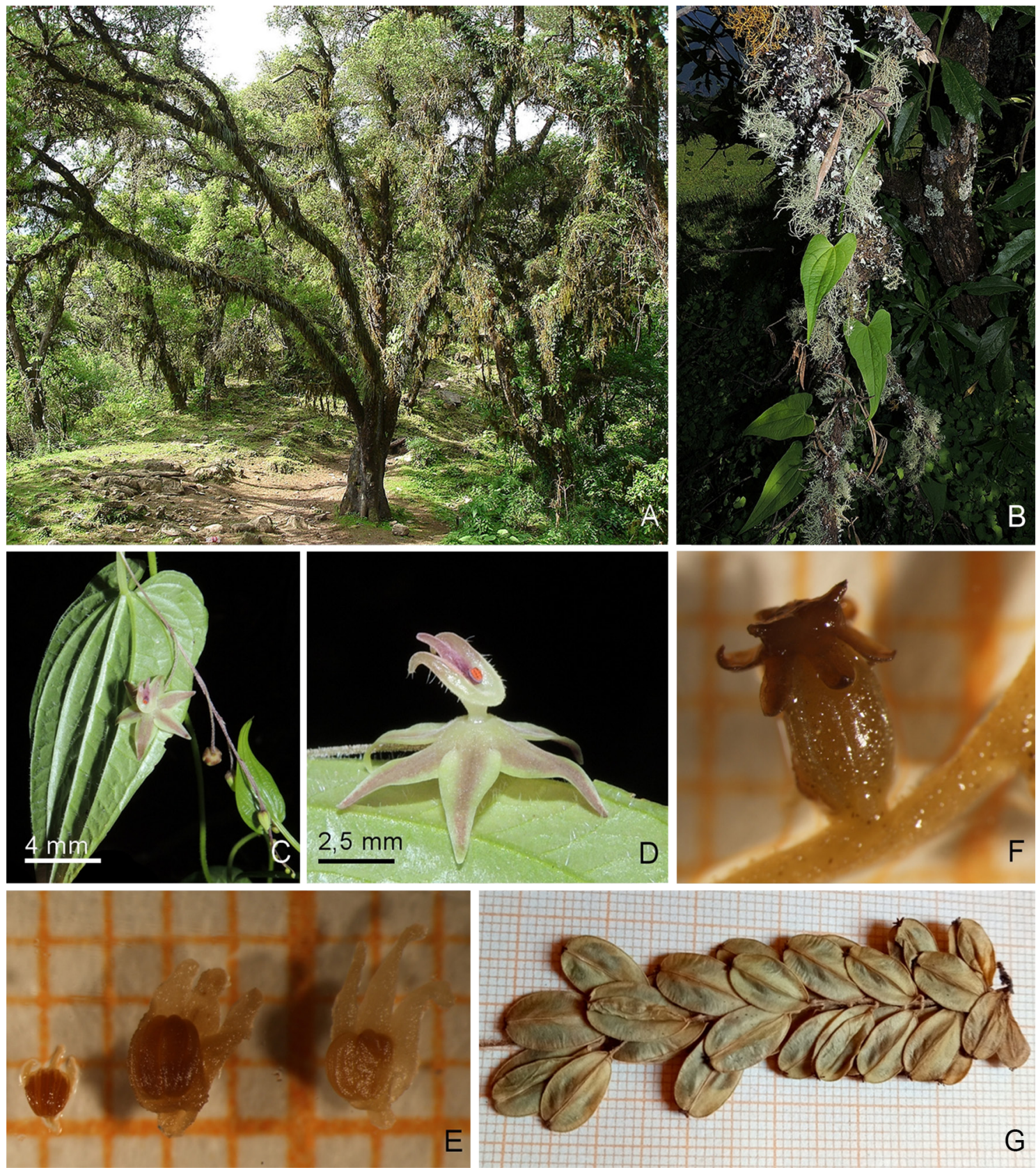

Fig. 3. Dioscorea ayardei. A, hábitat. B, hábito. C, flor estaminada. D, detalle del cuerpo estaminal. E, desarrollo del cuerpo estaminal. F, flor pistilada. G, frutos. Figura en color en la versión en línea http://www.ojs.darwin.edu.ar/ index.php/darwiniana/article/view/985/1239

Dioscorea glomerulata Hauman y D. cienegensis R. Knuth (Dioscoreaceae), Polylepis ssp. (Rosaceae), entre otras.
Fenología. Florece desde mediados de enero hasta marzo y fructifica desde marzo hasta abril. 
Tabla 1. Diferencias morfológicas entre $D$. ayardei y las especies relacionadas.

\begin{tabular}{|c|c|c|c|c|c|c|c|}
\hline & D. ayardei & D. cienegensis & D. haumanii & D. demourae & D. bermejensis & D. spectabilis & D. monandra \\
\hline $\begin{array}{c}\text { Hábitat y } \\
\text { Distribución }\end{array}$ & $\begin{array}{l}\text { Yungas en } \\
\text { Bosque } \\
\text { Montano } \\
\text { superior. } \\
\text { Endémica de } \\
\text { Argentina }\end{array}$ & $\begin{array}{l}\text { Yungas en Selva } \\
\text { Basal y Bosque } \\
\text { Montano inferior } \\
\text { en Argentina, } \\
\text { Bosque Atlántico } \\
\text { en Brasil }\end{array}$ & $\begin{array}{l}\text { Yungas en } \\
\text { Selva Basal } \\
\text { y Bosque } \\
\text { Chaqueño. } \\
\text { Argentina }\end{array}$ & $\begin{array}{l}\text { Selva Paranaense } \\
\text { en Argentina. } \\
\text { Cerrado, Bosque } \\
\text { Atlántico, } \\
\text { Pampa en Brasil }\end{array}$ & $\begin{array}{l}\text { Yungas en } \\
\text { Bosque } \\
\text { Montano } \\
\text { superior. } \\
\text { Argentina }\end{array}$ & $\begin{array}{l}\text { Yungas en Bosque } \\
\text { Montano superior } \\
\text { en Argentina, } \\
\text { Yungas Peruano- } \\
\text { Bolivianas en } \\
\text { Bolivia }\end{array}$ & $\begin{array}{l}\text { Yungas en } \\
\text { Bosque } \\
\text { Montano. } \\
\text { Argentina }\end{array}$ \\
\hline $\begin{array}{c}\text { Inflorescencia } \\
\text { masculina: } \\
\text { longitud }\end{array}$ & $5-10 \mathrm{~cm}$ & $5-10 \mathrm{~cm}$ & $8-13 \mathrm{~cm}$ & $8-17 \mathrm{~cm}$ & $3-4 \mathrm{~cm}$ & $10-12 \mathrm{~cm}$ & $4-5 \mathrm{~cm}$ \\
\hline $\begin{array}{c}\text { Flor } \\
\text { estaminada: } \\
\text { diámetro }\end{array}$ & $10-13 \mathrm{~mm}$ & $14-16 \mathrm{~mm}$ & $10-14 \mathrm{~mm}$ & $14-20 \mathrm{~mm}$ & 6-10 mm & $16-24 \mathrm{~mm}$ & $5-7 \mathrm{~mm}$ \\
\hline $\begin{array}{l}\text { Perianto flor } \\
\text { estaminada: } \\
\text { forma }\end{array}$ & Estrellado & Rotáceo & Rotáceo & Rotáceo & Rotáceo & Rotáceo & Campanulado \\
\hline Tépalos: color & $\begin{array}{l}\text { Purpúreo en } \\
\text { el centro con } \\
\text { bordes blanco- } \\
\text { verdosos }\end{array}$ & $\begin{array}{l}\text { Verdoso a } \\
\text { violáceo }\end{array}$ & Purpúreo & $\begin{array}{l}\text { Verdoso a } \\
\text { purpúreo }\end{array}$ & $\begin{array}{l}\text { Verdoso a } \\
\text { levemente } \\
\text { purpúreo }\end{array}$ & $\begin{array}{l}\text { Blanco verdoso } \\
\text { a violáceo }\end{array}$ & $\begin{array}{l}\text { Blanco } \\
\text { amarillento }\end{array}$ \\
\hline $\begin{array}{l}\text { Tricomas en } \\
\text { la columna } \\
\text { estaminal }\end{array}$ & Presente & Ausente & Presente & Presente & Ausente & Presente & Ausente \\
\hline $\begin{array}{c}\text { Anteras: } \\
\text { posición respecto } \\
\text { de la columna } \\
\text { estaminal }\end{array}$ & $\begin{array}{l}\text { Inserta en el } \\
\text { centro }\end{array}$ & $\begin{array}{l}\text { Insertas en la } \\
\text { porción media }\end{array}$ & $\begin{array}{l}\text { Insertas en la } \\
\text { porción basal, } \\
\text { entre las } \\
\text { proyecciones }\end{array}$ & $\begin{array}{l}\text { Insertas en los } \\
\text { ángulos apicales }\end{array}$ & $\begin{array}{l}\text { Insertas en la } \\
\text { porción media }\end{array}$ & $\begin{array}{l}\text { Insertas en la } \\
\text { base de las } \\
\text { proyecciones }\end{array}$ & $\begin{array}{l}\text { Inserta en el } \\
\text { ápice }\end{array}$ \\
\hline $\begin{array}{l}\text { Anteras: } \\
\text { cantidad }\end{array}$ & Una & Tres & Tres & Tres & Tres & Tres & Una \\
\hline
\end{tabular}

Estado de conservación. Hasta el momento solo se conoce una pequeña población de D. ayardei, que se mantuvo estable a lo largo de 10 años de muestreos y muy cercana al área protegida Parque Nacional Calilegua. Las principales amenazas que presenta la especie son el pisoteo y consumo por parte del ganado, así como el efecto de incendios debido a prácticas culturales en la región. Según los criterios de la IUCN $(2012,2017)$, esta especie puede ser considerada como Vulnerable (VU-D2). Consideramos necesario una evaluación formal a futuro que permita corroborar la categoría del estado de conservación sugerido.

\section{Observaciones taxonómicas}

1. En el año 1916 Hauman describió a Dioscorea monandra, especie que se caracteriza por la presencia de una única antera. El autor consideró que la columna estaminal se originó de la unión de los tres estambres, por lo que la propuso como especie tipo de una nueva sección: Monandria.
Años más tarde Knuth (1924), en su obra sobre la Familia Dioscoreaceae, posicionó la especie de Hauman dentro de la sección Monadelpha Uline. Couto (2015) por su parte, teniendo en cuenta la diagnosis de dicha sección ampliada más tarde por Knuth (1924) (las especies de esta sección poseen tres estambres unidos en una columna estaminal cilíndrica o en un cuerpo carnoso ovoide) sumado a las características de su especie tipo, D. monadelpha (Kunth) Griseb., sostuvo que D. monandra no presentaba las características típicas de la sección y se la debía mantener dentro de la sección propuesta por Hauman. Sin embargo, recientes estudios filogenéticos de Viruel et al. (2018) y Couto et al. (2018) ubican a D. monandra cercana a las especies de la sección Monadelpha. Se necesita estudiar la filogenia de $D$. ayardei y comprobar su cercanía con D. monandra y con las especies afines para aportar una nueva sistematización. Además, es necesario realizar un estudio detallado sobre la ontogenia de la columna estaminal en la sección Monadelpha. 
2. Del análisis de los protólogos y de imágenes de alta resolución de los ejemplares tipo de las especies afines, surge que $D$. ayardei es afín a $D$. haumanii Xifreda, pero difiere por las características de las flores masculinas: perigonio patente (vs. perigonio reflexo), cuerpo estaminal de forma navicular (vs. cuerpo estaminal ovoide) con tres proyecciones dendriformes casi horizontales (vs. 3, corniculiformes laminares, erectas, curvadas en la parte superior y bordes pilosos) y una antera inserta en el centro de la estructura (vs. 3, alternas con las estructuras carnosas).

\section{Material estudiado (Paratipos)}

Dioscorea ayardei. ARGENTINA. Jujuy. Depto. Valle Grande, Sierras de Calilegua, camino al Alto de Calilegua, pasando Quebrada Honda, 25-II-2009, (さ), Bulacio \& Ayarde 1055 (LIL); Puesto Aguas Blancas, 12-I-2017, (ð), Bulacio \& Ayarde 3170 (LIL); Puesto Duraznillo, 24-II2015, (ð゚ㅇ), Asesor et al. 115 (LIL); Quebrada de Cortaderas, 26-II-2016, (ぷ+ + ), Bulacio \& Ayarde 3029 (LIL); Quebrada por Paso Despensa, 29-III2009, (ぷ+o), Bulacio \& Ayarde 1288 (LIL).

\section{Material adicional estudiado}

Dioscorea bermejensis R. Knuth. ARGENTINA. Salta. Depto. Santa Victoria, Los Toldos, 2200 m s.m., IV-1903, (ठ), Fiebrig 3249 (Holotipo B).

Dioscorea cienegensis R. Knuth. ARGENTINA. Salta. III-1873, ( $\overbrace{}^{\Uparrow})$, Hieronymus \& Lorentz s.n. (Lectotipo B). Depto. La Caldera, Quebrada de Gallinato, 1100-1200 m s.m., 1-IV2001, (ठ), Tolaba 2882 (SI). Tucumán. Depto. Burruyacú, Sierras de Medina, 23-III-2017, (ð), Asesor \& Bulacio 202 (LIL).

Dioscorea demourae R. Knuth. BRASIL. Ceará. Fortaleza, Praia Grande, 24-I-2012, (す), Verdi et al. 2611 (FURB). Rio de Janeiro, Theresopolis, s.d., (ठ), De Moura 535 (Holotipo B). Santa Catarina. Itapiranga, hacia el rio Peperiguassu, 15-II-1934, ( ${ }^{\Uparrow}$ 우), Rambo 1473 (LIL).

Dioscorea haumanii Xifreda. ARGENTINA. Salta. Depto. Anta, 3-IV-1971, (ð̊ㅇ), Saravia et al. 146 (LIL). Depto. Cerrillos, 1150 m s.m., 11XI-1990, (ð゚ㅇ) Novara \& Bruno 9527 (MCNS). Depto. Orán, Urundel, arroyo Lipán, 12-V-1942, (ठ), Hunziker 2023 (Isotipo LIL).
Dioscorea monandra Hauman. ARGENTINA. Jujuy. Depto. Capital, Yala, 13-I-1951, (ð゚ㅇ), Meyer 16984 (LIL). Tucumán. Depto. Tafí del Valle, La Peñita, 26-XII-1907, (ð̊ำ), Castillón 584 (Sintipo LIL).

Dioscorea spectabilis R. Knuth. ARGENTINA. Jujuy. Depto. Ledesma, Abra de Cañas, 10IV-1971, (ठ), Legname \& Cuezzo 4490 (LIL). Depto. Valle Grande, Sierras de Calilegua, camino a Alto Calilegua, 24-II-2015, (ð゚+ Asesor et al. 129 (LIL).

BOLIVIA. Cochabamba. Prov. Chapare, Espíritu Santo, VI-1911, (ふ), Herzog 2264 (Holotipo L, Isotipo B).

\section{CONCLUSIONES}

El hallazgo de $D$. ayardei eleva a 8 el número de especies de Dioscorea para las Sierras de Calilegua y a 34 para el país. Además, reafirmamos la necesidad de encarar el estudio filogenético de la nueva especie para verificar su cercanía con $D$. monandra y su ubicación en la sección correspondiente. Asimismo, es necesario estudiar la ontogenia de la columna estaminal de las especies de la sección Monadelpha así como de las que poseen un único estambre para determinar su relación sistemática.

\section{AGRADECIMIENTOS}

Agradecemos a la Fundación Miguel Lillo por la financiación de los viajes de campo y equipamiento necesario utilizados para concretar esta investigación, que se enmarca en el proyecto B-0022-1 (Miguel Lillo 251, T4000JFE, Tucumán). A Lelia Bordón de la sección Iconografía (FML), por la realización de las láminas de este trabajo. A Manuel Emiliano González Arévalo y Asunción Cano Echeverria del Herbario San Marcos y al curador del Herbario del Instituto Científico Michael Owen Dillon (Perú). A Alejandro Araujo Murakami y Marisol Toledo del Herbario del Oriente Boliviano. A Magaly Mercado del Herbario del Centro de Biodiversidad y Genética de Cochabamba, Bolivia. A Carla Maldonado y Alfredo Fuentes del Herbario Nacional de Bolivia. 


\section{BIBLIOGRAFÍA}

Asesor, P. N.; P. L. Albornoz \& E. Bulacio. 2019. Evidencias del origen de una posible nueva entidad de Dioscorea (Dioscoreaceae) de las Sierras de Calilegua, Jujuy (Argentina). Un enfoque morfo-anatómico. Lilloa 56(2): 1-17. DOI: https://doi.org/10.30550/j.li1/2019.56.2/1

Ayarde, H. R. 2013. Aporte hídrico de las neblinas en las montañas húmedas del noroeste de Argentina. Actas del I Simposio de la Reserva de Biosfera de las Yungas y V Reunión Regional de las Selvas de Montañas, San Salvador de Jujuy, Argentina, Tomo I: 1-13.

Bulacio, E. \& H. R. Ayarde. 2009. Flora y Vegetación del sector alto de las Sierras de Calilegua (Jujuy). XXXII Jornadas Argentinas de Botánica, Boletín de la Sociedad Argentina de Botánica, Córdoba, Argentina, 44(supl.): 106.

Cabrera, A. L. 1976. Regiones fitogeográficas argentinas. Enciclopedia argentina de agricultura y jardinería, tomo 2, fasc. 2. Buenos Aires: Acme.

Coursey, D. G. 1967. Yams; an account of the nature, origins, cultivation and utilisation of the useful members of the Dioscoreaceae. London: Longmans.

Couto, R. S. 2015. Filogenia de Dioscorea subgênero Helmia (Dioscoreaceae) com ênfase no neotrópico e revisão de Dioscorea seção Monadelpha. Rio de Janeiro (Brasil): Tese Doutorado, Museu Nacional/Universidade Federal do Rio de Janeiro.

Couto, R. S.; A. C. Martins, M. Bolson, R. C. Lópes, E. Smidt \& J. M. Braga. 2018. Time calibrated tree of Dioscorea (Dioscoreaceae) indicates four origins of yams in the Neotropics since the Eocene. Botanical Journal of the Linnean Society 188(2): 144-160. DOI: https://doi. org/10.1093/botlinnean/boy052

Hauman, L. 1916. Les Dioscoréacées de L'Argentine. Anales del Museo Nacional de Historia Natural de Buenos Aires 27: 441-513.
IUCN. 2012. Categorías y criterios de la lista roja de la Unión International para la Conservación de la Naturaleza UICN. Versión 3.1. Gland, Suiza. Segunda edición. https:// www.iucn.org/es/content/categorias-y-criterios-de-lalista-roja-de-la-uicn-version-31-segunda-edicion

IUCN Standards and Petitions Subcommittee. 2017. Guidelines for Using the IUCN Red List Categories and Criteria. Version 14. Prepared by the Standards and Petitions Subcommittee. Disponible en www.iucnredlist. org/documents/RedListGuidelines.pdf

Knuth, R. 1924. Dioscoreaceae. En A. Engler, Pflanzenreich $I V$, pp. 1-387. Berlín: Cramer Verlag.

Morello, J.; S. D. Matteucci, A. F. Rodríguez, M. Silva \& C. J. de Haro. 2012. Ecorregiones y complejos ecosistémicos argentinos. Buenos Aires: Orientación Gráfica Editora.

Thiers, B. [permanently updated, accessed 2021]. Index Herbariorum: a global directory of public herbaria and associated staff. New York Botanical Garden's Virtual Herbarium, http://sweetgum.nybg.org/ih/

Viruel, J.; F. Forest, O. Paun, M. Chase, D. Devoy, R. S. Couto, J. G. Segarra-Mragues, P. Catalán \& P. Wilkin. 2018. A nuclear Xdh phylogenetic analysis of yams (Dioscorea: Dioscoreaceae) congruent with plastid trees reveals a new Neotropical lineage. Botanical Journal of the Linnean Society 187(2): 232-246. DOI: https://doi.org/10.1093/ botlinnean/boy013

Wilkin, P.; P. Schols, M. W. Chase, K. Chayamarit, C. A. Furness, S. Huysmans, F. Rakotonasolo, E. Smets \& C. Thapyai. 2005. A plastid gene phylogeny of the yam genus, Dioscorea: roots, fruits and Madagascar. Systematic Botany 30: 736-749. DOI: https://doi. org/10.1600/036364405775097879

Zuloaga, F. O.; O. Morrone \& M. J. Belgrano (eds.), Dioscorea (Dioscoreaceae). Catálogo de las plantas vasculares de Argentina. Instituto de Botánica Darwinion. on-line http:// www.floraargentina.edu.ar. Acceso junio 2021. 